\title{
SELF-SENSING POSITION DETERMINATION ON A SENSOR-DESIGNED PROPORTIONAL SOLENOID
}

\author{
Thomas Kramer*, Jürgen Weber \\ Chair of Fluid-Mechatronic Systems, Institute of Mechatronic Engineering, Technische Universität Dresden, \\ Helmholtzstrasse 7a, 01069 Dresden \\ * Corresponding author: Tel.: +49 351463 31964; E-mail address: thomas.kramer@tu-dresden.de
}

\begin{abstract}
Proportional valves are widely used in fluid systems for controlling the volume flow rate or fluid pressure. The actuation of this valves is done by PWM-driven proportional solenoids, which enable self-sensing position determination abilities due to air gap-dependent electrical behaviour, e. $\mathrm{g}$. for condition monitoring or position controlling tasks. However, the sensor properties of conventional proportional solenoids are poor due to ambiguities caused by hysteresis effects (magnetic hysteresis, eddy currents) and saturation effects. Thus, a sensor-designed actuator was developed with very low hysteresis effects and unique position determination by using electrical sheet and a particular air gap design. This paper deals with investigations of a novel self-sensing position determination approach on a demonstrator of the sensor-designed solenoid. The advantage of this method is an online consideration of transient effects such as mean current change and armature motion as well as temperature-dependent resistance. For this, a combined evaluation of the differential inductance and flux linkage during PWM periods is proposed.
\end{abstract}

Keywords: self-sensing position determination, proportional solenoid, sensor design

\section{INTRODUCTION}

Proportional solenoids are well-known drives for proportional valves in order to control the volume flow rate or fluid pressure in a continuous manner. The working principle is based on the reluctance principle. By exciting the solenoid's coil, a magnetic field arises and a magnetic force $F_{\mathrm{m}}$ acts on the movable armature (Figure 1). The magnetic force works against a spring force $F_{\mathrm{S}}$ for setting a specific position according to the equilibrium of the forces. A linear behaviour between the electrical input (coil current $i$ ) and mechanical output (armature position $x$ ) is achieved with a particular air gap design and a magnetic excitation in the saturation area. A cone shaped counterpart of the armature results in a nearly position-independent magnetic force [1]. The excitation in the saturation area leads to a current proportional magnetic force [2]. The coil is usually excited by an energy efficient PWM voltage $u_{\text {in }}$ to avoid static friction.

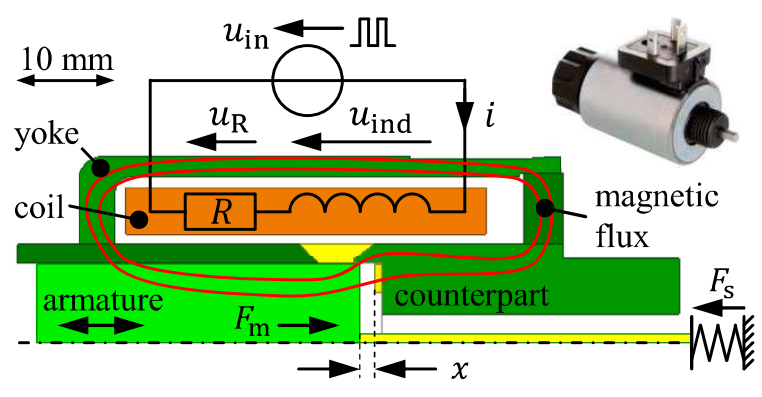

Figure 1: Design of conventional proportional solenoid (axisymmetrical magnetic circuit; without fluid stage)

It is well-known that this electro-magnetomechanical operating chain is not reactionless. The magnetic behaviour is air gap-dependent and has an influence on the electrical side due to its inductive effect. This behaviour can be exploited to get information about the armature position only by measuring electrical values such as current and voltage. So-called self-sensing position determination methods can be used for various applications, e.g. for condition monitoring [3] or position controlling tasks [4]. 
However, the sensor properties of conventional proportional solenoids are poor due to ambiguities caused by hysteresis effects (magnetic hysteresis, eddy currents) and saturation effects. Reason for this is the focus on the actuator capability in nowadays development activities of conventional actuators. Thus, a sensor-designed actuator was developed with very low hysteresis effects and unique position determination by using electrical sheet and a particular air gap design.

The focus of this paper is the investigation of a novel self-sensing position determination approach on the developed sensor-designed solenoid. Firstly, some basics of the position observation and the sensor properties of conventional proportional solenoids are shown. Based on this, a demonstrator of the sensordesigned proportional solenoid and its electromagnetic behavior is presented. In the main part, a promising self-sensing position determination method is described and extended according to considering transient effects such as mean current change and armature motion. The coil resistance is an important value for position observation methods. An online capable method for calculating the resistance is presented. After that, the used test rig for investigating the methods is described. Finally, the achieved results of position observation and resistance determination applied on the demonstrator are presented.

\section{SELF-SENSING POSITION DETERMINATION}

As described above, the air gap-dependent magnetic behaviour can be used for self-sensing position determination. There are several methods known from literature. Richter gives an overview in [5] and [6], especially for application at typical valve's solenoids. The most promising methods for PWM-driven proportional solenoids are the evaluation of the differential inductance [7] and differential current change [8]. These methods work with the existing PWM excitation and do not need any additional test signal. Furthermore, they are not based on numerical integration such as flux linkage method [4][9], which is especially difficult in the continuous working principle of proportional solenoids. In this work, only the differential inductance method is considered, because the differential current change is only a consequence of the position-dependent inductance.

The differential inductance $L_{\mathrm{d}}$ is derived from flux linkage $\Psi$ according to

$L_{\mathrm{d}}=\frac{\mathrm{d} \Psi}{\mathrm{d} i}$.

The flux linkage is the magnetic flux summed over all windings. It can be calculated by integrating the induced voltage $u_{\text {ind }}$ according to the induction law with

$$
\begin{array}{rl}
\Psi=\int u_{\text {ind }} \mathrm{d} & t+\Psi_{0} \\
=\int\left(u_{\text {in }}-R i\right) \mathrm{d} t+\Psi_{0}
\end{array}
$$

The induced voltage is calculated from measured coil current $i$ and input voltage $u_{\text {in }}$ (see Figure 1). Thus, the differential inductance is calculated according to

$$
L_{\mathrm{d}}=\frac{u_{\mathrm{in}}-R i}{\frac{\mathrm{d} i}{\mathrm{~d} t}}
$$

without any numerical integration.

\section{SENSOR PROPERTIES OF CONVENTIONAL PROPORTIONAL SOLENOIDS}

Generally, existing proportional solenoids are developed considering only the actuator properties, while sensor behaviour is not included in the developing process. In order to investigate the current sensor properties, the electromagnetic behaviour has to be analysed. The evaluation of flux linkage $\Psi(i, x)$ maps all relevant effects. From this, the characteristic map of differential inductance $L_{\mathrm{d}}(i, x)$ is derived, which is necessary for self-sensing position determination. The maps are generated from measurements of coil current $i$ and input voltage $u_{\text {in }}$ in steady state (Figure 2). Therefore, the armature is fixed at various positions $x$ and the solenoid is excited by a PWM voltage with a constant duty cycle $d$ (PWM period: $T_{\mathrm{PWM}}$ ). The measurements are evaluated as soon as the mean current value does not change anymore. The map values are calculated from the measurements according to equations (2) and (3), here by neglecting the unknown start value $\Psi_{0}$ (remanent flux linkage) in equation (2). 


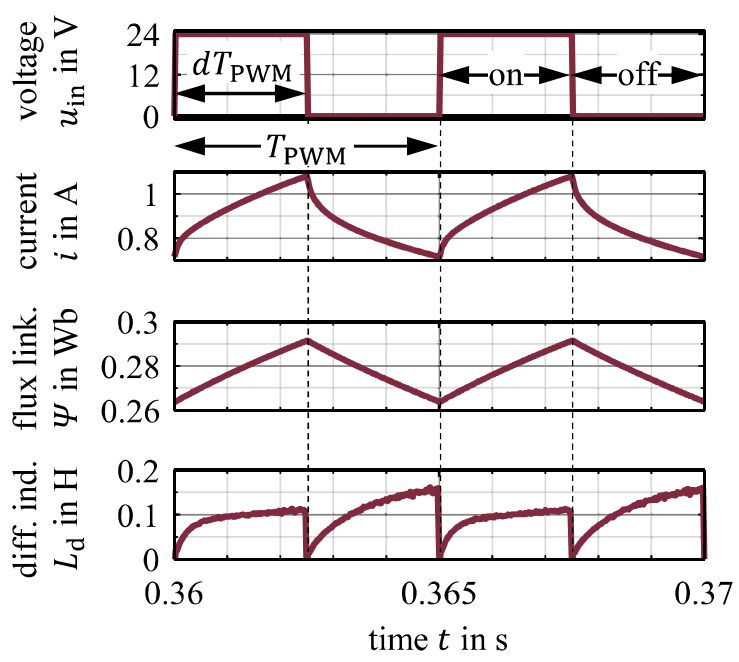

Figure 2: Time-dependent measurements of electrical values in steady state and map values calculated therefrom

Figure 3 illustrates both maps depending on coil current $i$ at various PWM duty cycles $d$ and armature positions $x$.
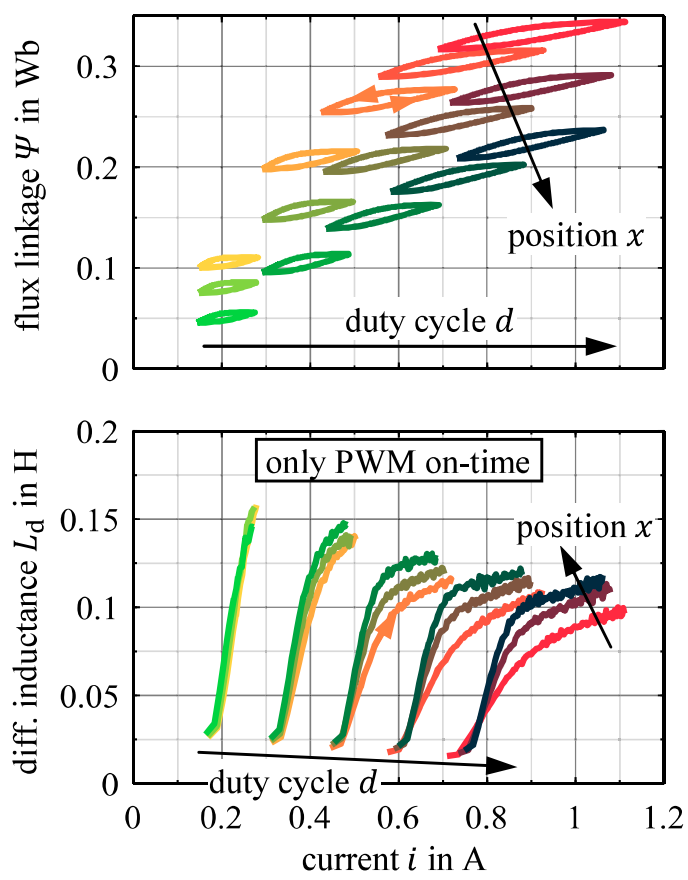

Figure 3: Map of flux linkage $\Psi(i, x)$ and differential inductance $L_{\mathrm{d}}(i, x)$ of a conventional proportional solenoid calculated from measurements (in operating range)

Two main effects can be derived from the behaviour. Firstly, there are striking hysteresis loops during PWM excitation (Figure 3, top), which represent the inductive energy losses due to magnetic hysteresis and eddy currents. In relation to the position observation, they lead to ambiguities in position determination (Figure 3, bottom), since a clear assignment of the evaluated differential inductance to exactly one position is unfeasible. This problem becomes even greater, when transient processes are considered (armature motion, change of mean current). The second effect becomes visible, when hysteresis effects are not taken into account (Figure 4). The so-called anhysteretic curves are derived from FE simulations. They show nearly no position dependency in the range of larger currents due to saturation effects.

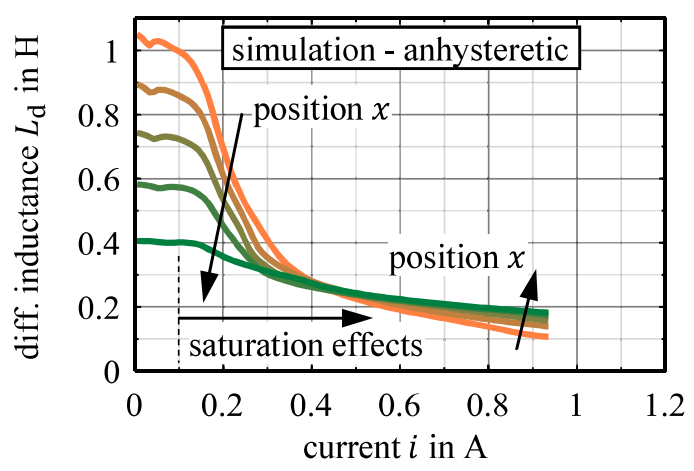

Figure 4: Map of differential inductance $L_{\mathrm{d}}(i, x)$ of a conventional proportional solenoid simulated without hysteresis effects (in operating range)

For these reasons, it was investigated how a proportional solenoid can be designed regarding proper sensor behaviour by considering the actuator characteristics as well. On this basis, a promising self-sensing design was built up as demonstrator. Detailed investigations for the development of sensor-designed solenoids will be published in a further article.

\section{SENSOR-DESIGNED PROPORTIONAL SOLENOID}

The aim of sensor-designed actuators is an unambiguous linear behaviour between the evaluated value for position observation (differential inductance) and the armature position. This basic characteristic can be achieved by a particular air gap design and an excitation of the magnetic circuit below saturation. In addition, the hysteresis effects have to be significantly reduced. The most promising method for lower hysteresis effects is the usage of electrical sheets. This material has a reduced 
magnetic hysteresis and avoids large eddy currents due to its isolated sheets. An excitation below saturation and a lamination of the magnetic circuit was already proposed in [10].

Figure 5 shows the developed demonstrator design of a sensor-designed proportional solenoid in size comparison to the conventional solenoid. The increased actuator size is necessary for achieving the same force level by considering an excitation below saturation.
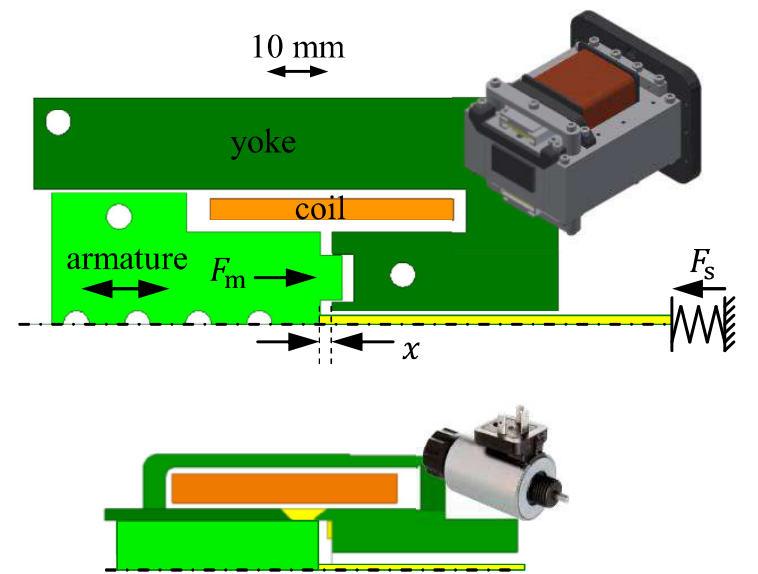

Figure 5: Design of sensor-designed proportional solenoid (planar magnetic circuit, top) in size comparison to the conventional solenoid (bottom)
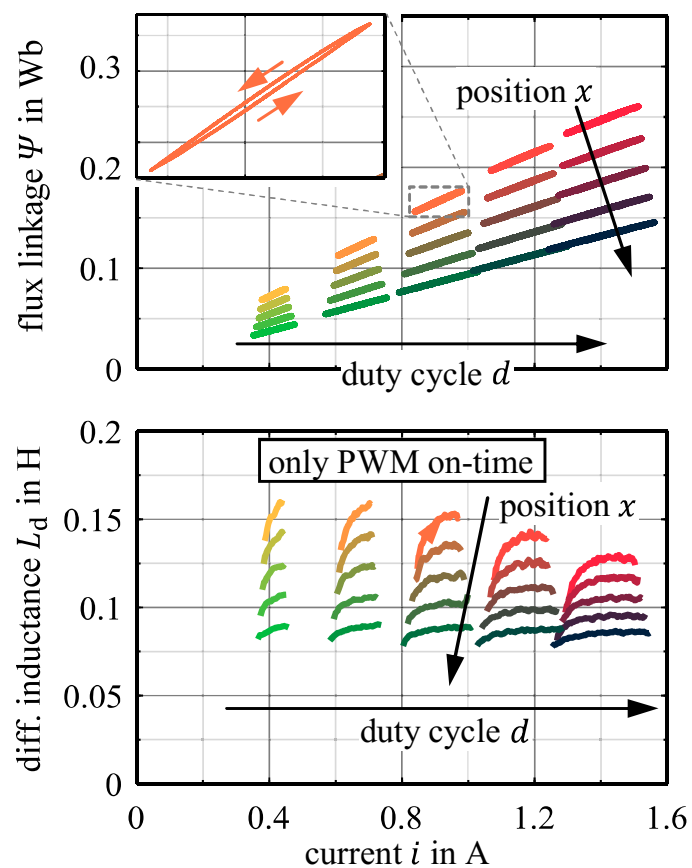

Figure 6: Map of flux linkage $\Psi(i, x)$ and differential inductance $L_{\mathrm{d}}(i, x)$ of the developed sensordesigned solenoid calculated from measurements (in operating range)
The magnetic behaviour was measured and simulated in the same way as on the conventional proportional solenoid. The improved magnetic behaviour is illustrated in Figure 6 and Figure 7.

Compared to the behaviour of conventional solenoids presented in Figure 3, a significant reduction of the hysteresis losses can be seen (Figure 6, top). However, small loops still occur due to remaining small magnetic hysteresis effects and eddy currents. This results in a varying differential inductance during PWM ontime respectively off-time (Figure 6, bottom), which requires a special handling.

Furthermore, the linear position-dependent behaviour of the differential inductance, which is derived from FE simulations, is pronounced over the entire operating range (Figure 7). Slight saturation effects occur at large currents. This is due to a compromise between actuator size and sensor properties.

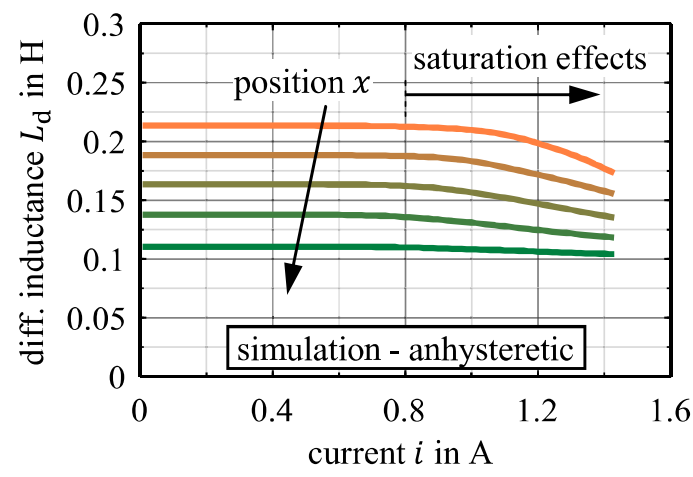

Figure 7: Map of differential inductance $L_{\mathrm{d}}(i, x)$ of the developed sensor-designed solenoid simulated without hysteresis effects (in operating range)

\section{POSITION OBSERVATION STRATEGY FOR SENSOR-DESIGNED SOLENOID}

\subsection{Position observation}

The remaining hysteresis effects can be handled by a mean value strategy. This is allowable due to nearly linear curves in the map $\Psi(i, x)$ and triangular current behaviour (Figure 8). Therefore, the averaged differential inductance is calculated from the current and voltage values according to Figure 8 with

$L_{\mathrm{d}, \mathrm{on}}=\frac{u_{\mathrm{in}, \mathrm{on}}-R i_{\mathrm{on}}}{\frac{\Delta i_{\mathrm{on}}}{d T_{\mathrm{PWM}}}}$. 
for PWM on-time and with

$$
L_{\mathrm{d}, \mathrm{off}}=\frac{u_{\mathrm{in}, \mathrm{off}}-R i_{\mathrm{off}}}{\frac{\Delta i_{\mathrm{off}}}{(1-d) T_{\mathrm{PWM}}}} .
$$

for PWM off-time.

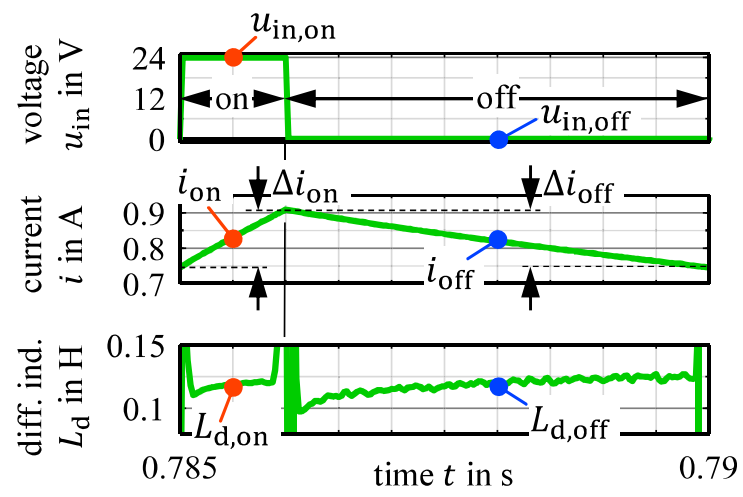

Figure 8: Measured and calculated values for mean value strategy

By using this mean value strategy, the resulting map has a clear correlation, shown in Figure 9. Exactly one position is assigned to a defined pair of differential inductance and current value. Due to nearly same inductance values $L_{\mathrm{d} \text {,on }}$ and $L_{\mathrm{d} \text {,off }}$, the averaged value $L_{\mathrm{d}, \mathrm{ss}}$ of both is used in the following (ss: steady state).

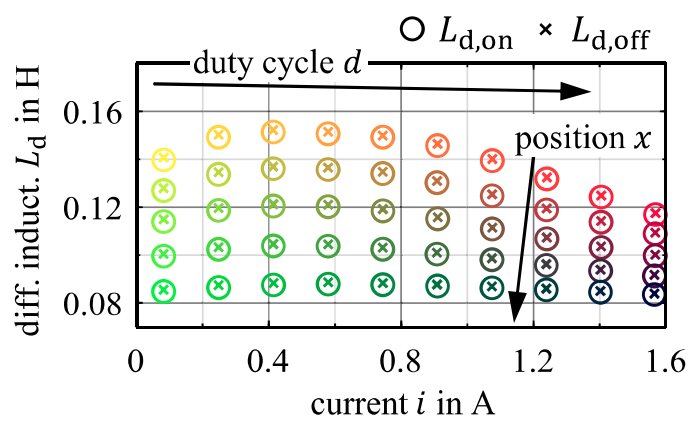

Figure 9: Map of differential inductances $L_{\mathrm{d}, \mathrm{on}}\left(i_{\mathrm{on}}, x\right)$ and $L_{\mathrm{d}, \mathrm{off}}\left(i_{\mathrm{off}}, x\right)$ in operating range by applying mean value strategy (unipolar PWM voltage)

For these investigations, a unipolar PWM voltage was used (on-time: $24 \mathrm{~V}$, off-time: $0 \mathrm{~V}$ ). As the duty cycle is zero $(d=0$, zero mean current), a position determination is not possible. However, a bipolar PWM voltage can overcome this issue and will be used in the following (on-time: $24 \mathrm{~V}$, off-time: $-24 \mathrm{~V}$ ). The corresponding map is illustrated in Figure $\mathbf{1 0}$ and shows the feasibility at zero mean current.

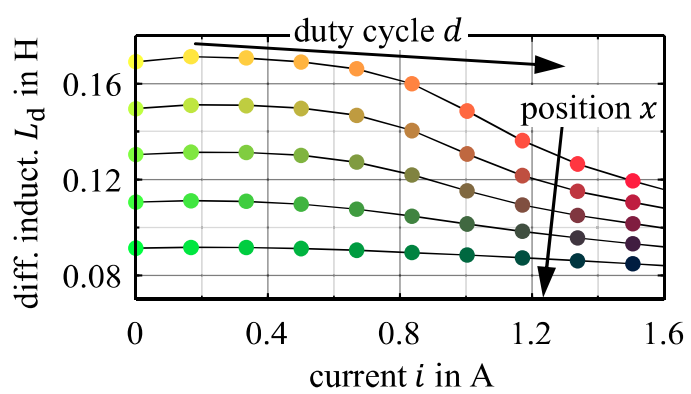

Figure 10: Map of differential inductances $L_{\mathrm{d}, s \mathrm{~s}}(i, x)$ in operating range by applying mean value strategy (bipolar PWM voltage)

The described method works for steady state without changing the mean current value and without armature motion. If there is a change of the mean current, the hysteresis loops are not closed. This results in different inductance values $L_{\mathrm{d}, \text { on }}$ and $L_{\mathrm{d}, \mathrm{off}}$ as for steady state $\left(L_{\mathrm{d}, \mathrm{ss}}\right)$ due to transient behaviour. Figure 11 depicts the behaviour schematically.
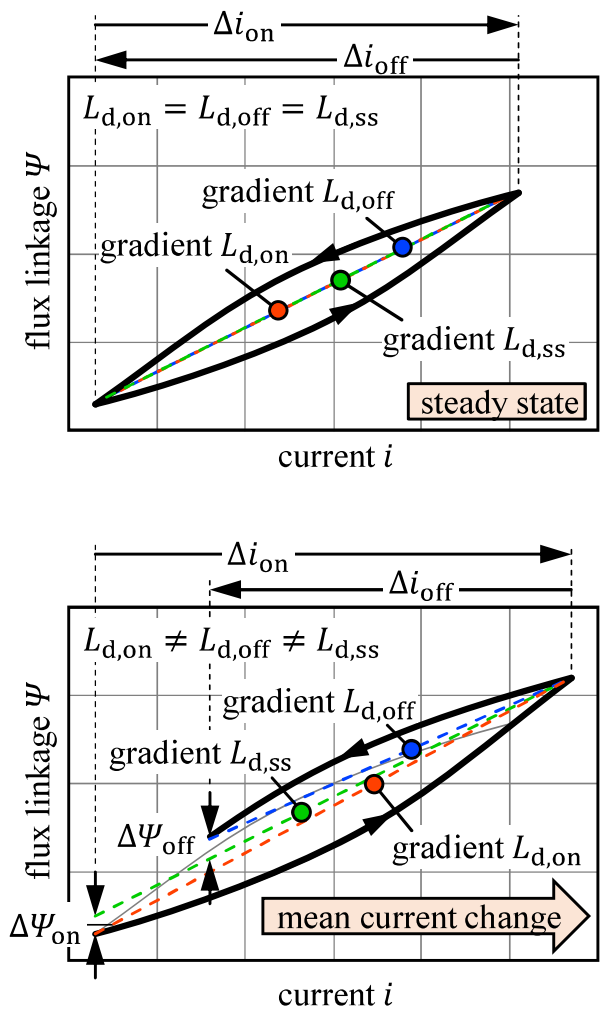

Figure 11: Schematic behaviour of flux linkage over current during a PWM period (top: at steady state, bottom: at change of mean current)

A change of the duty cycle leads to a transient phenomenon of the mean current value. If the duty cycle is increased, the mean current increases successively as well. Therefore, the 
bent flux linkage curve at PWM on-time increases further than in the comparable operating point of steady state. This results in a larger gradient $L_{\mathrm{d}, \text { on }}$ (Figure 11, bottom). During PWM off-time, the curve is shortened with a resulting smaller gradient $L_{\mathrm{d}, \text { off }}$. A similar behaviour occurs during armature motion due to the position-dependent change of flux linkage (Figure 12). If the air gap decreases due to armature motion, the flux linkage increases and vice versa (compare also Figure 6, top: positiondependency of flux linkage).

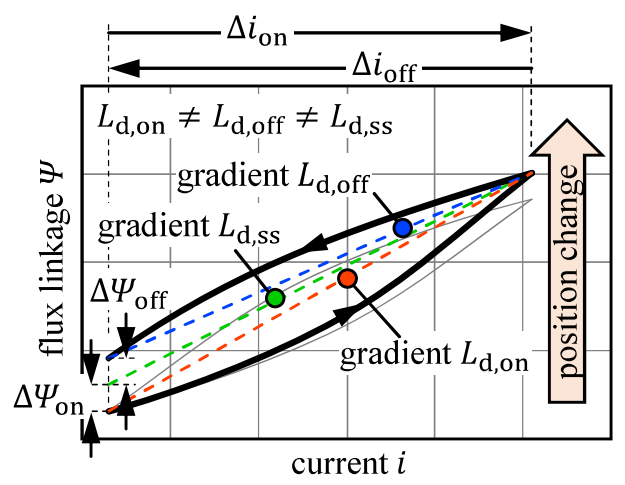

Figure 12: Schematic behaviour of flux linkage over current during a PWM period at armature motion

However, a real armature movement is initiated by a change of the mean current. Therefore, the effects from mean current change (Figure 11, bottom) and armature motion (Figure 12) are superimposed.

Both effects can be considered by a correction term. The induction law (see equation (2)) provides an appropriate approach. Therefore, the Kirchhoff's voltage law for the electrical domain can be written as

$u_{\text {in }}=R i+u_{\text {ind }}=R i+\frac{\mathrm{d} \Psi(i, x)}{\mathrm{d} t}$

with a current- and position-dependent flux linkage $\Psi(i, x)$. The partial derivation of the flux linkage results in

$u_{\mathrm{in}}=R i+\frac{\mathrm{d} \Psi(i, x)}{\mathrm{d} i} \frac{\mathrm{d} i}{\mathrm{~d} t}+\frac{\mathrm{d} \Psi(i, x)}{\mathrm{d} x} \frac{\mathrm{d} x}{\mathrm{~d} t}$.

The last term describes already the influence of the armature motion, but can consider a change of the mean current due to its similar behaviour as well. By using equation (1) for the second term and defining the third term as a general induced voltage correction term, equation (7) can be written as $u_{\text {in }}=R i+L_{\mathrm{d}} \frac{\mathrm{d} i}{\mathrm{~d} t}+\frac{\mathrm{d} \Psi_{\text {cor }}}{\mathrm{d} t}$.

The application of equation (8) to PWM ontime and off-time by considering the mean value strategy results in

$u_{\text {in,on }}=R i_{\text {on }}+L_{\mathrm{d}, \text { on }} \frac{\Delta i_{\text {on }}}{d T_{\mathrm{PWM}}}+\frac{\Delta \Psi_{\mathrm{on}}}{d T_{\mathrm{PWM}}}$

and

$$
\begin{aligned}
u_{\text {in }, \text { off }}=R i_{\text {off }} & +L_{\mathrm{d}, \text { off }} \frac{\Delta i_{\text {off }}}{(1-d) T_{\mathrm{PWM}}} \\
& +\frac{\Delta \Psi_{\mathrm{off}}}{(1-d) T_{\mathrm{PWM}}} .
\end{aligned}
$$

The flux linkage values $\Delta \Psi_{\text {on }}$ and $\Delta \Psi_{\text {off }}$ are the deviations over PWM on-time respectively offtime compared to steady state behaviour (see also Figure 11, bottom and Figure 12). These values can be calculated by numerical integration according to equation (2). The integration is possible here, since the integration time is short (maximum: $T_{\mathrm{PWM}}$ ) and only the difference is calculated $\left(\Psi_{0}\right.$ not considered).

If the induced voltage correction is considered, the differential inductance values in equations (9) and (10) are without influences of armature motion and mean current change. It applies

$L_{\mathrm{d}, \mathrm{ss}}=L_{\mathrm{d}, \mathrm{on}}=L_{\mathrm{d}, \mathrm{off}}$.

The equations (9) to (11) yield the final calculation of differential inductance. By equating the coil resistance $R$, the differential inductance can be calculated with

$$
\begin{gathered}
L_{\mathrm{d}, \mathrm{ss}}=\frac{u_{\mathrm{in}, \mathrm{off}} i_{\text {on }}-u_{\mathrm{in}, \mathrm{on}} i_{\mathrm{off}}}{\frac{\Delta i_{\mathrm{off}}}{(1-d) T_{\mathrm{PWM}}} i_{\text {on }}-\frac{\Delta i_{\mathrm{on}}}{d T_{\mathrm{PWM}}} i_{\text {off }}} \\
+\frac{\frac{\Delta \Psi_{\mathrm{on}}}{d T_{\mathrm{PWM}}} i_{\text {off }}-\frac{\Delta \Psi_{\mathrm{off}}}{(1-d) T_{\mathrm{PWM}}} i_{\text {on }}}{\frac{\Delta i_{\text {off }}}{(1-d) T_{\mathrm{PWM}}} i_{\text {on }}-\frac{\Delta i_{\mathrm{on}}}{d T_{\mathrm{PWM}}} i_{\text {off }}} .
\end{gathered}
$$

The position can now be determined using this differential inductance value, the weighted mean current

$i_{\text {avg }}=d i_{\text {on }}+(1-d) i_{\text {off }}$

over a PWM period and the map from Figure 10.

\subsection{Resistance determination}

The calculations of the flux linkage values $\Delta \Psi_{\text {on }}$ and $\Delta \Psi_{\text {off }}$ require the actual value of the coil 
resistance according to (2). Unfortunately, the resistance value is temperature-dependent and has to be determined online for a correct calculation. There are some methods known from literature (e. g. [11][12][13]), which are mostly complex or are not accurate enough. A simple approach is an averaging over one PWM period according to

$R_{\mathrm{avg}}=\left.\frac{\overline{u_{\mathrm{in}}}}{\bar{i}}\right|_{T_{\mathrm{PWM}}}$.

Another approach is equating the differential inductance according to equation (9) and (10) [14]. It must be taken into account that the calculation of the correction terms (similar to equation (12)) itself depends on the resistance. In order to avoid numerical loops, the correction terms are negligible, and the resistance value can be calculated with

$$
\begin{aligned}
& R_{\text {eq }} \\
& =\frac{u_{\text {in,off }} \frac{\Delta i_{\text {on }}}{d T_{\mathrm{PWM}}}-u_{\text {in,on }} \frac{\Delta i_{\text {off }}}{(1-d) T_{\mathrm{PWM}}}}{\frac{\Delta i_{\text {on }}}{d T_{\mathrm{PWM}}} i_{\text {off }}-\frac{\Delta i_{\text {off }}}{(1-d) T_{\mathrm{PWM}}} i_{\text {on }}} .
\end{aligned}
$$

Both methods work well at steady state, but result in deviations during armature motion and change of the mean current. The results can be improved by using low pass filtering (similar to [11]). This is possible due to very different time constants between the slow thermal behavior of the resistance change and the fast-acting armature motion as well as a change of the mean current. The filtered resistance is calculated by

$$
R_{\mathrm{f}, k}=\frac{R_{\mathrm{avg} / \mathrm{eq}}+\frac{\tau}{T_{\mathrm{PWM}}} R_{\mathrm{f}, k-1}}{1+\frac{\tau}{T_{\mathrm{PWM}}}},
$$

where $R_{\text {avg/eq }}$ is the calculated resistance according to equation (14) or (15), $R_{\mathrm{f}, k-1}$ is the previous calculated filtered value and $R_{\mathrm{f}, k}$ the new filtered value. The time constant $\tau$ is an adjustable parameter and defines the filter characteristic.

\section{TEST RIG}

The above described methods were investigated by using the test rig, which is seen in Figure 13.

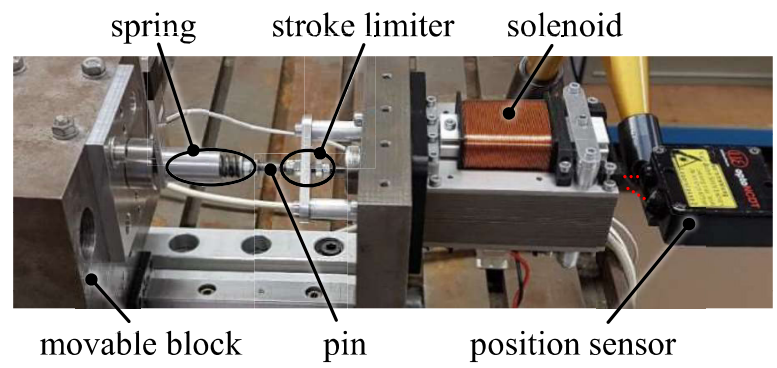

Figure 13: Test rig for investigations of the sensordesigned proportional solenoid (here: configuration for armature motion with spring)

The steady state behavior is measured at fixed armature position. Therefore, the movable block is directly connected to the pin (without spring). Pin and armature are force-fit linked. Various air gap values are set with the block position. The actual armature position is measured with a position sensor (laser triangulation).

The behavior during mean current changes is measured with the same configuration.

For measurements of armature motion, the armature respectively pin works against a spring (see Figure 13). In order to move the armature only in the position's operating range, a stroke limiter is used.

During all measurements, coil current, input voltage and armature position are recorded by a LabVIEW-based cRio-System. All used analogue digital converters have a resolution of 16 bit. The time resolution is $25 \mu \mathrm{s}$. Based on the measured values, the described methods for position observation and resistance determination are investigated.

\section{RESULTS}

The above developed novel method for selfsensing position determination is investigated step by step. Therefore, results are shown for steady state and transient behavior. Firstly, the accuracy at steady state is determined. Thus, the repeatability of the map operating points as well as intermediate operating points are investigated. Figure 14 depicts the different operating points in relation to the map points. 


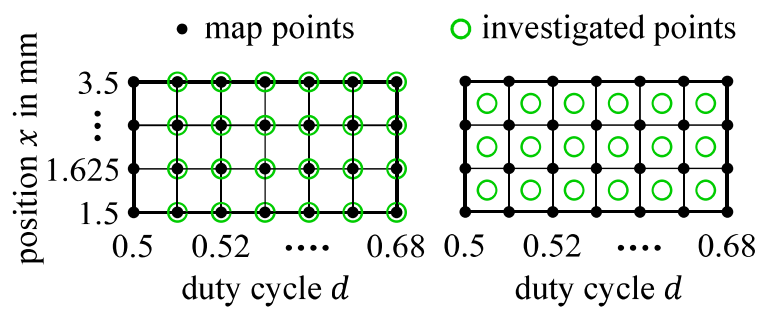

Figure 14: Operating points for investigations in steady state related to the map points (left: repeatability of map points, right: accuracy of intermediate operating points)

The results are illustrated in Figure 15. Therefore, the various operating points were analysed once. The relative differences between the measured position value $x_{\mathrm{m}}$ (from position sensor) and observed position value $x_{\mathrm{ss}}$ in relation to the operating range $\Delta x_{\mathrm{op}}=2 \mathrm{~mm}$ are evaluated according to

$\Delta x_{\mathrm{r}}=\frac{x_{\mathrm{m}}-x_{\mathrm{ss}}}{\Delta x_{\mathrm{op}}}$.

The induced voltage correction is not considered due to steady state investigation.
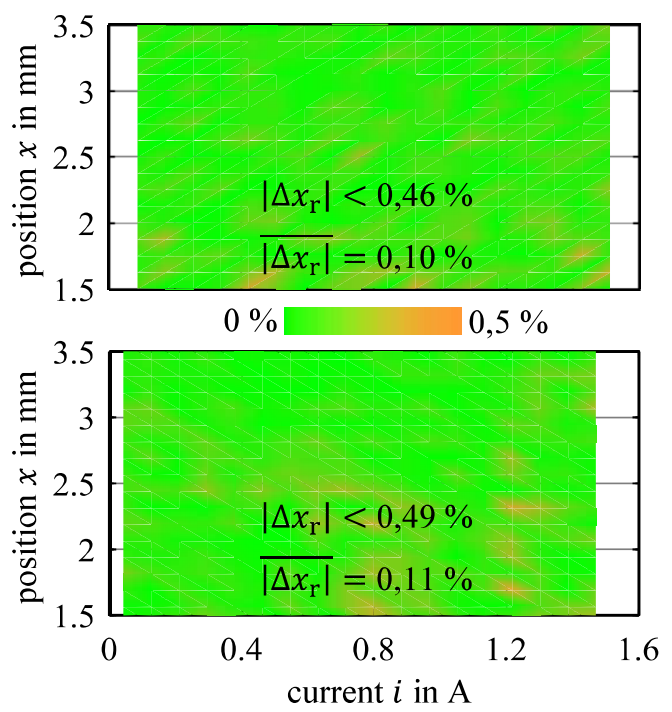

Figure 15: Accuracy of position observation at steady state (top: repeatability of map points, bottom: accuracy of intermediate points)

The plots show very good matches between position observation and position measurement over the entire operating range. Reasons for the small deviations may include interpolation during map generation and position observation as well as measurement noise.
Secondly, the position observation at a mean current change and fixed armature position is investigated. Therefore, Figure 16 depicts the current response as well as measured and calculated position values after a duty cycle step. The mean current change at beginning can be read from the current curve. Consequently, the magnetic force arises and leads to a small movement due to the elasticity of the test rig. The position observation without considering the correction terms ( $x_{\text {on }}$ and $x_{\text {off }}$ by using equations (4) and (5)) illustrates the deviations during the mean current change. The extended method with induced voltage correction enables an improved position determination, here with good matches as well $\left(\Delta x_{\mathrm{r}, \mathrm{cor}}<1 \%\right)$. All calculations are based on the steady state resistance value $R_{\text {avg }}$ according to equation (14).

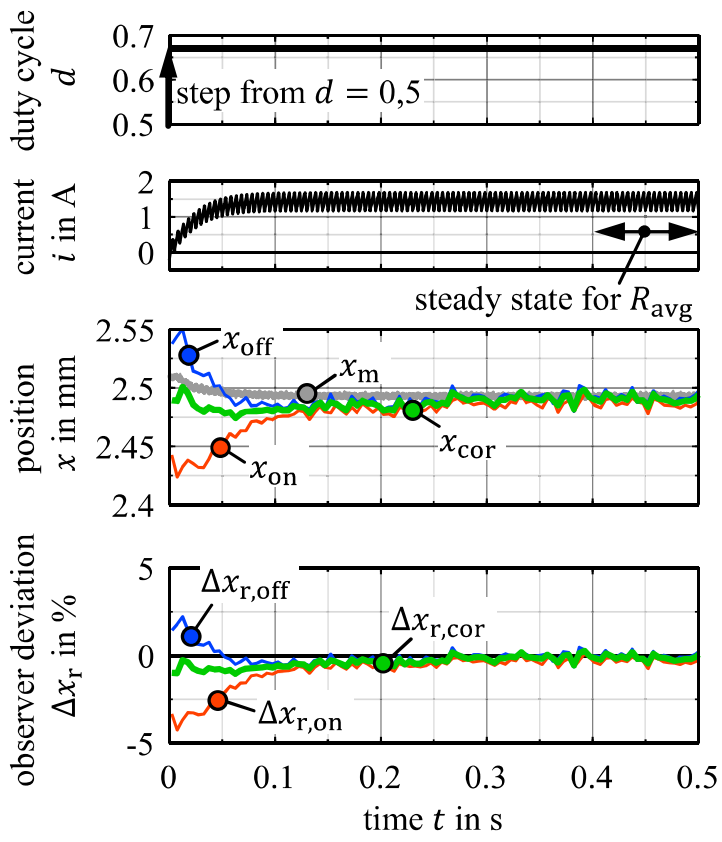

Figure 16: Position observation at change of mean current due to duty cycle step, without and with considering induced voltage correction

In the third step, the position observation method is analysed at armature motion. Therefore, a duty cycle step initiates the position change and a second step back leads to the return motion. The step's edge time $t_{\text {edge }}$ is adjustable. The results are shown in Figure 17. 

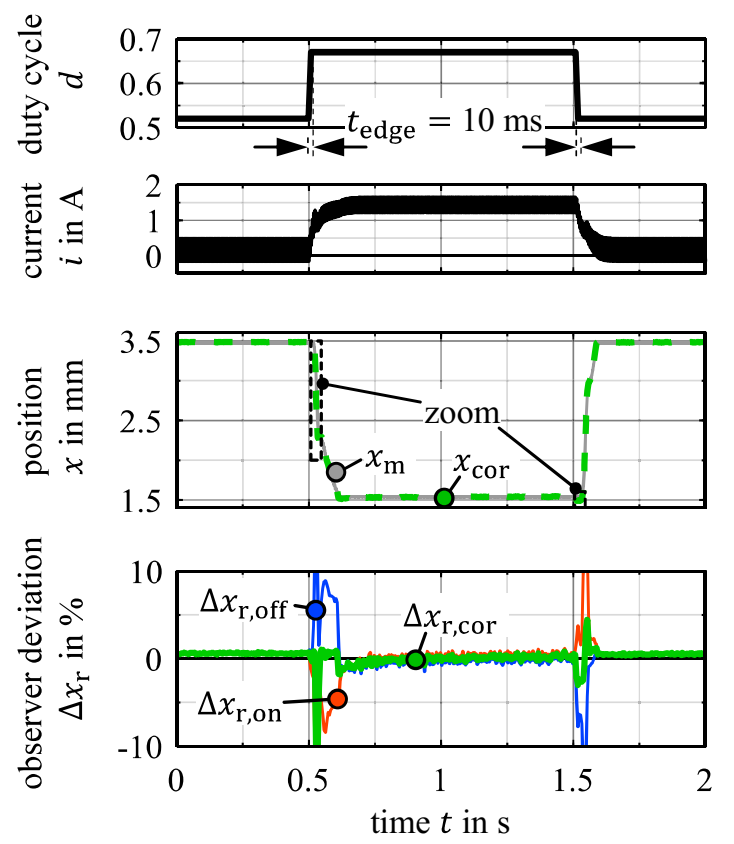

Figure 17: Position observation at fast armature motion due to duty cycle step, without and with considering induced voltage correction

Here, again, the non-consideration of the correction terms causes deviations in the selfsensing position determination. The observation is significantly improved by using the novel method. Large differences still occur at fast armature motion (Figure 18). These can be caused by dynamic effects at the test rig and by the mean value based observation method. However, the maximum deviation is only about half the distance the armature moves during a $\mathrm{PWM}$ period $T_{\mathrm{PWM}}=5 \mathrm{~ms}$.

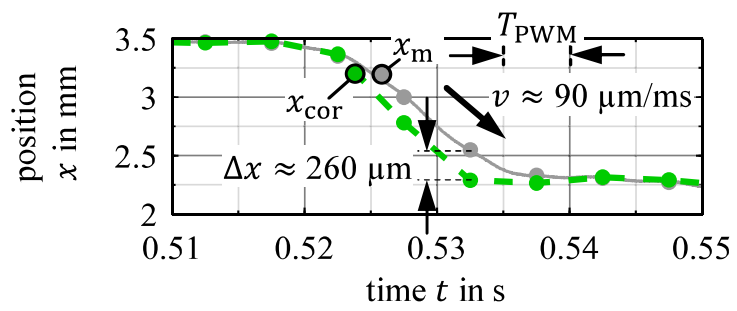

Figure 18: Zoomed behaviour of measured and observed position during armature motion

Further deviations occur at high mean current gradient, especially at high currents and small air gaps (Figure 19). In this area, the map of the differential inductance has a pronounced nonlinear current dependency due to saturation effects (Figure 20). This results in deviations when using the averaging method, which is based on linear interpolation.

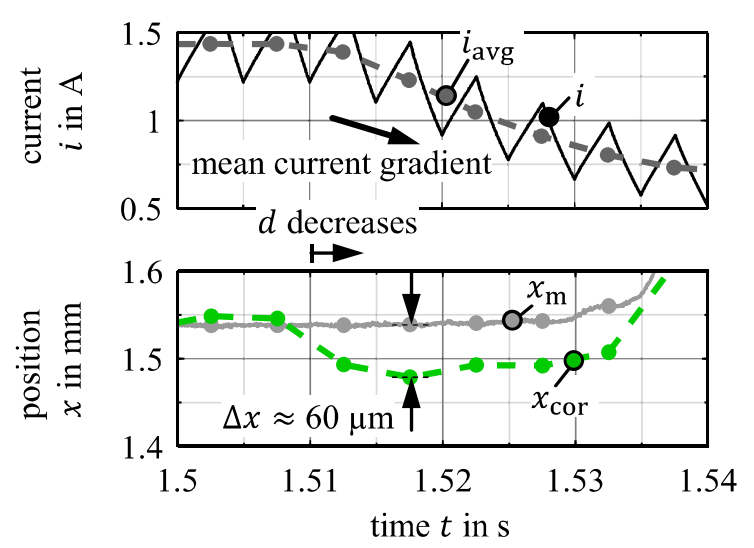

Figure 19: Zoomed behaviour of current as well as measured and observed position during mean current gradient

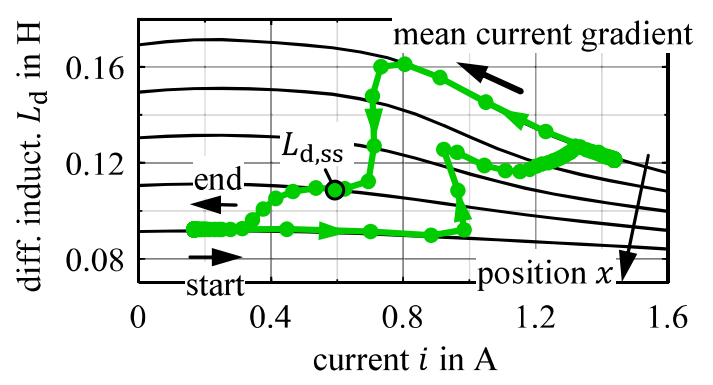

Figure 20: Behaviour of the time-dependent calculated differential inductance over the measuring time in the map

An increased PWM frequency can reduce the deviations. But it must be taken into account that the resolution of one PWM-period and of the current difference $\Delta i$ decreases.

The above shown investigation uses the resistance value from steady state according to equation (14). This method is not online capable. Therefore, the proposed methods for resistance determination are analysed in the following. Figure 21 illustrates the calculated resistance values for the same control regime as in Figure 17. The basis is the reference value $R_{\text {ref }}$, which is obtained from the averaged resistance value $R_{\text {avg }}$ in steady state (same as used for previous investigation). 

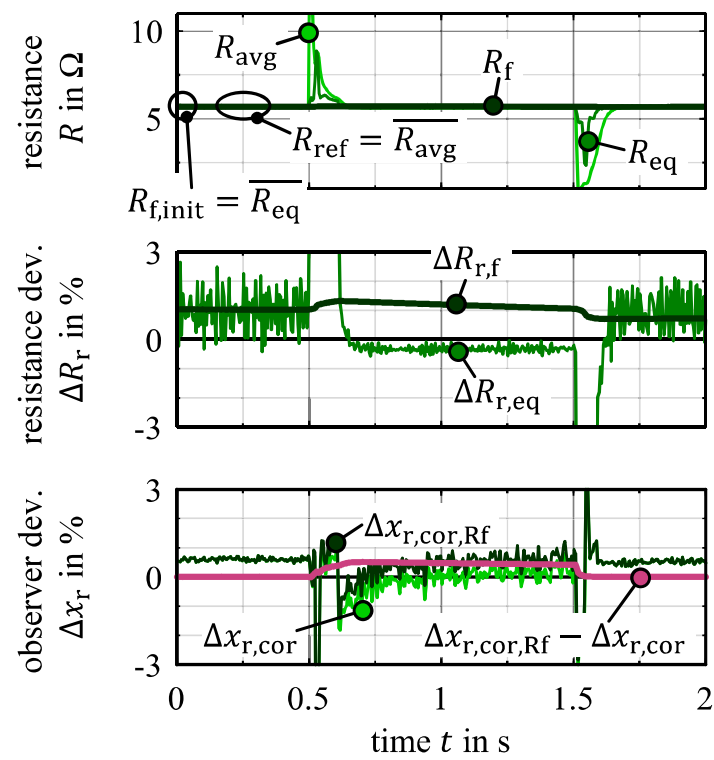

Figure 21: Resistance determination methods at armature motion and resulting observer deviation

As expected, deviations occur at armature motion respectively mean current change. By applying the described filter, the calculated resistance value can be smoothed. Therefore, the proposed equating method with $R_{\mathrm{eq}}$ (equation (15)) is filtered due to its smaller deviation in contrast to the averaged method with $R_{\text {avg }}$ (equation (14), see Figure 21, top). The filter parameter is set to $\tau=5 \mathrm{~s}$ and the mean of the first five calculations of $R_{\mathrm{eq}}$ is used to initiate the filter $\left(R_{\mathrm{f}, \text { init }}\right)$. Based on the relative resistance deviation

$\Delta R_{\mathrm{r}, \mathrm{f}}=\frac{R_{\mathrm{f}}-R_{\mathrm{ref}}}{R_{\mathrm{ref}}}$,

the working principle of the filter can be seen. At transient effects, the filtered resistance value changes only slightly. An armature motion in one direction results in a slight increased resistance value, while the return motion leads to a decreased resistance value. During steady state, the resistance value $R_{\mathrm{f}}$ aligns slowly to $R_{\text {eq }}$.

The resulting difference of the position observation between both methods ( $R_{\text {ref }} \rightarrow \Delta x_{\mathrm{r}, \text { cor }}$ and $R_{\mathrm{f}} \rightarrow \Delta x_{\mathrm{r}, \text { cor, Rf }}$ ) is in the order of magnitude of resistance deviation $\Delta R_{\mathrm{r}, \mathrm{f}}$ (ca. $1 \%$ ).

In order to analyse the real online capability of this method, further works are planned. Especially longer durations have to be investigated, where the resistance changes significantly due to thermal effects.

The position observation works with further control regimes as well. Exemplary, Figure 22 shows a second investigation. The armature does not move between the stroke limits, but has a smaller stroke. In addition, the step's edge time is set to $t_{\text {edge }}=300 \mathrm{~ms}$.
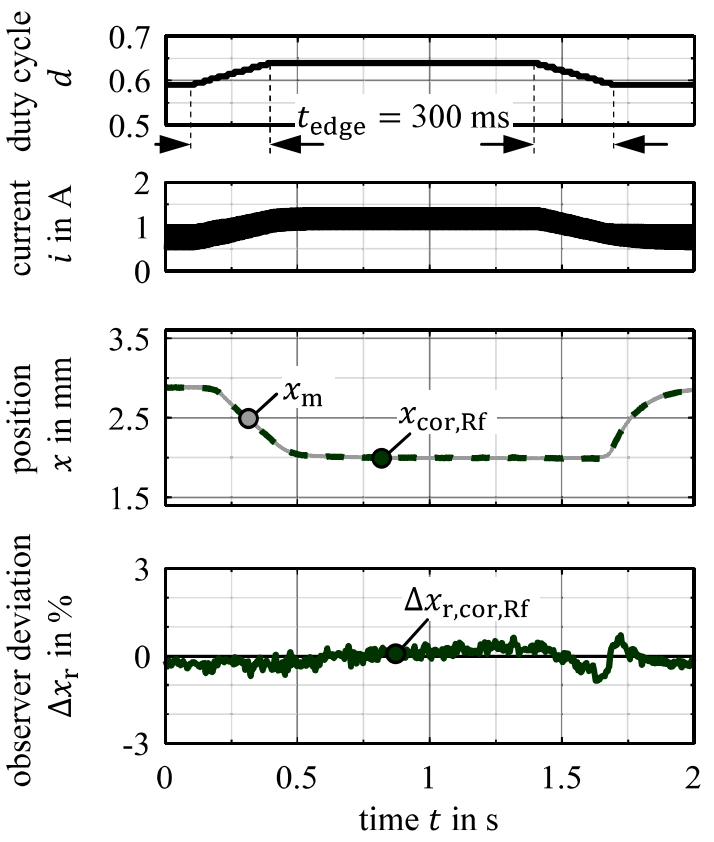

Figure 22: Position observation at slow armature motion due to duty cycle step, only with considering induced voltage correction

The results show a similar behaviour. Due to slower armature motion, a smooth behaviour without deviation peaks can be seen.

\section{CONCLUSION AND OUTLOOK}

In this work, a novel self-sensing position determination method for a sensor-designed proportional solenoid is proposed. This method considers in addition to steady state behaviour also transient effects such as mean current change and armature motion. It is based on a combined evaluation of differential inductance and flux linkage.

Firstly, issues of conventional proportional solenoids concerning sensor behavior are described. These are mainly hysteresis and saturation effects. On this basis, a sensordesigned proportional solenoid is proposed, which exhibits improved sensor characteristics due to a particular air gap design and a magnetic 
circuit made from electrical sheets. Because of remaining small hysteresis effects, a mean value strategy is proposed for self-sensing position determination. The basic position observation uses the differential inductance method. In order to consider also transient effects, the method is extended by an induced voltage correction, which is based on the evaluation of the flux linkage. Furthermore, the position determination method requires the coil resistance value, which must be known online. Different simple methods for calculating the coil resistance are described. Finally, investigations at a demonstrator of the sensor-designed solenoid are presented. The analyses show promising results. The position observation can be significantly improved by applying the novel observation method. Remaining deviations occur especially at high armature velocities and partly at high mean current gradients.

The method is appropriate for condition monitoring the armature position and simple position control tasks, applied on similar working solenoids as well. For a more precise control, further investigations have to be done especially concerning higher resolution of time and analogue digital converter. For an application as valve drive, the design has to be adapted.

Further works will deal with investigations of the resistance determination method at longer evaluation durations, where the resistance value changes significantly due to thermal effects. Therefore, it is also planned to implement the entire observation method and the solenoid control into electronics, in order to show potential industrial usability.

\section{ACKNOWLEDGEMENT}

The research presented in this paper is based on the project "Sensorgerechte Konstruktion elektromagnetischer Ventilaktoren" (Ref.-No. AiF 19093 BR/1), which was financed and supervised by the Research Association Mechanical Engineering (FKM). In the scope of the Programme to promote Industrial Collective Research it was funded by the German Federation of Industrial Research Associations (AiF) with means of the

\section{Supported by:}

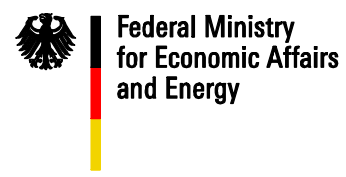

on the basis of a decision by the German Bundestag
Federal Ministry of Economic Affairs and Energy (BMWi) on the basis of a decision by the German Bundestag.

$\begin{array}{ll}\text { NOMENCLATURE } \\ d & \text { duty cycle (-) } \\ F & \text { force }(\mathrm{N}) \\ i & \text { current }(\mathrm{A}) \\ L & \text { inductance }(\mathrm{H}) \\ R & \text { resistance }(\Omega) \\ t & \text { time }(\mathrm{s}) \\ T & \text { time period }(\mathrm{s}) \\ u & \text { voltage }(\mathrm{V}) \\ v & \text { velocity }(\mathrm{m} / \mathrm{s}) \\ x & \text { air gap, position }(\mathrm{m}) \\ \tau & \text { time constant }(\mathrm{s}) \\ \Psi & \text { flux linkage }(\mathrm{Wb})\end{array}$

\section{REFERENCES}

[1] Kallenbach E, Eick R, Ströhla T, Feindt K, Kallenbach M, Radler O (2018): Elektromagnete - Grundlagen, Berechnung, Entwurf und Anwendung. 5th edition, Springer Vieweg, Wiesbaden, Germany, 2018

[2] Kramer T, Weber J (2019), Sensorgerechte Konstruktion elektromagnetischer Ventilaktoren, Final report, AiF 19093 BR/1, Dresden, Germany, September 27, 2019

[3] Kramer T, Weber J, Pflug G, Harnisch B (2018), Intelligent Condition Monitoring of Bi-stable Process Valves. 11th International Fluid Power Conference, IFK 2018, Aachen, Germany, March 19-21, 2018

[4] Kramer T, Petzold M, Weber J, Ohligschläger O, Müller A (2016), Smart control of electromagnetically driven dosing pumps. 10th International Fluid Power Conference, IFK 2016, Dresden, Germany, March 8-10, 2016

[5] Richter S, Weber J (2013), Sicherheitsorientierte Zustandsüberwachung an elektromagnetisch betätigten Ventilen. Informationsveranstaltung des Forschungsfonds des Fachverbandes Fluidtechnik im VDMA, Frankfurt/Main, Germany, June 26, 2013

[6] Richter S (2016), Ein Beitrag zur Integration von Maßnahmen der Funktionalen Sicherheit in einen geregelten elektrohydraulischen Antrieb für stationäre Anwendungen. Dissertation, Technische Universität Dresden, Dresden, Germany, 2016 
[7] Rahman M F, Cheung N C, Lim K W (1995), Position Estimation in Solenoids Actuators. Conference Record of the 1995 IEEE Industry Applications Conference Thirtieth IAS Annual Meeting, IAS 1995, Orlando, Florida, USA, October 8-12, 1995

[8] Renn J C, Chou Y S (2004), Sensorless Plunger Position Control for a Switching Solenoid. JSME International Journal Series C47, June, 2004

[9] Kallenbach M (2005), Entwurf von magnetischen Mini- und Mikroaktoren mit stark nichtlinearem Magnetkreis. Dissertation, Technische Universität Ilmenau, Ilmenau, Germany, 2005

[10] Maridor J (2011), Design, Optimization, and Sensorless Control of a Linear Actuator. Dissertation, École Polytechnique Fédérale de Lausanne, Lausanne, Switzerland, 2011
[11] Glück T, Kemmetmüller W, Tump C, A Kugi (2010), Resistance estimation algorithm for selfsensing magnetic levitation systems. 5th IFAC Symposium on Mechatronic Systems, Cambridge, Massachusetts, USA, September 1315,2010

[12] Jung H G, Hwang J Y, Yoon P J, Kim J H (2007), Resistance Estimation of a PWM-driven solenoid. International Journal of Automotive Technology, Vol. 8, No. 2, pp. 249-258, 2007

[13] Dülk I, Kovácsházy T (2015), Parameter Estimation in Linear Electromagnetic Devices. IEEE Transaction on Industrial Electronics, Vol. 62, No. 6, June, 2015

[14] Kramer T, Weber J, Pflug G, Harnisch B (2018), Smart Realisation of Energy Savings during Switching Operation at Bi-stable Process Valves. Actuator 2018 - 16th International Conference on New Actuators, Bremen, Germany, June 25-27, 2018 